\title{
Fine needle aspiration cytology in the diagnosis of multiple myeloma
}

\author{
Luiz Antonio Guimarães Cabral ${ }^{1}$, Andrea Silveira Penteado ${ }^{2}$, \\ Adriana Aigotti Haberbeck Brandão ${ }^{1}$, Janete Dias Almeida ${ }^{1^{*}}$ \\ ${ }^{1}$ Department of Biosciences and Oral Diagnosis, São José dos Campos Dental School, UNESP_Univ Estadual Paulista, São José dos \\ Campos, São Paulo, Brazil; *Corresponding Author: janete@fosjc.unesp.br \\ ${ }^{2}$ Private Practice, Canção Nova, Cachoeira Paulista, SP, Brazil
}

Received 3 October 2011; revised 3 November 2011; accepted 20 November 2011

\begin{abstract}
Multiple Myeloma (MM) is a disseminated plasma cell tumor caused by the proliferation of a single plasma cell clone which results from the production of monoclonal immunoglobulin, most commonly class $\mathbf{G}$ (IgG). MM is a cancer of the elderly, with a mean age at diagnosis of 68 years. A 79-year-old white patient sought the outpatient clinic of the Discipline of Stomatology, São José dos Campos Dental School, UNESP, because of a fracture associated with an osteolytic lesion in the right ascending ramus of the mandible. In view of the clinical-radiographic findings, exploratory fine needle aspiration (FNA) of the lesion was performed and the sanguinolent material obtained was submitted to the preparations of smears for cytology analysis. The diagnosis of MM was based on laboratory and radiographic findings. FNA cytology analysis of the lesion permitted a diagnostic hypothesis of MM.
\end{abstract}

Keywords: Multiple Myeloma; Diagnostic Imaging; Cytology

\section{INTRODUCTION}

Multiple Myeloma (MM) is a disseminated plasma cell tumor caused by the proliferation of a single plasma cell clone which results from the production of monoclonal immunoglobulin, most commonly class G (IgG). This plasma cell clone proliferates in the bone marrow in an uncontrolled manner, resulting in bone resorption [1-3].

The course of MM is associated with different organ dysfunctions such as renal failure, anemia, hypercalcemia, deposition of amyloid substance characterizing excess of immunoglobulin light chains, a reduced immune response, coagulation disorders, recurrent infections, and phenomena related to blood hyperviscosity. Clinical repercussions are reflected in symptoms such as fatigue, fever, weight loss and intensive pain of variable duration, generally in the chest and spine [1,4].

The condition is rare in Asians but roughly twice as common in Afro-Caribbean ethnic groups compared with Caucasians.

Diagnosis is based on laboratory and radiographic findings and depends on three abnormal results: bone marrow containing more than $15 \%$ plasma cells (normally no more than $4 \%$ of the cells in the bone marrow are plasma cells); generalized osteopaenia and/or lytic bone lesions on plain film radiography; blood serum and/or urine containing an abnormal protein [5]. Osteolytic lesions with a "punched-out" appearance are observed on radiographs affecting various bones [2].

The apparent increase in incidence and mortality rates among patients with MM in many studies is due to improved case ascertainment, especially among the elderly. Authors address exposure to radiation from atomic bombs, therapeutic and diagnostic radiation, workers in the nuclear industry field and discuss agricultural occupations, exposure to benzene, petroleum products, and engine exhaust and other industrial exposures. Tobacco use, obesity, diet, and alcohol ingestion are all possible causes of MM [6].

The staging of MM is based on the serum concentration of haemoglobin, calcium and paraprotein, urinary Bence-Jones protein excretion and the number of skeletal lesions seen on plain radiographs [4]. Chemotherapy is the treatment of choice and is based on the use of various protocols, such as cyclophosphamide, vincristine, melphalan, and prednisone (CVMP) [7]. In view of the nature of the disease, the relief of symptoms is of the utmost importance in order to improve the patient's quality of life.

Achieving complete response or a major response is a key prognostic factor for improved survival in MM. As a treatment that results in high rates of major responses, 
high-dose chemotherapy plus autologous stem-cell transplantation (HDT-ASCT) remains the standard of care in eligible patients [8].

This article reports a case of Multiple Myeloma showing FNA cytology analysis.

\section{METHODS}

A 79-year-old white patient sought the outpatient clinic of the Discipline of Stomatology, São José dos Campos Dental School, UNESP-Univ Estadual Paulista, because of a fracture associated with an osteolytic lesion in the right ascending ramus of the mandible. Clinical examination showed asymptomatic enlargement of the right chin region in the absence of any phlogistic sign on the skin of the affected area. Upon anamnesis, the patient reported a 1.5-month history of intense pain in the region after sneezing. Orthopantomography revealed a transverse fracture in the median portion of the right ascending mandibular ramus, associated with a radiolucent lesion affecting the region of the retromolar trigonum, mandibular angle and inferior portion of the ascending ramus on the same side (Figure 1). In view of the clinical-radiographic findings, exploratory FNA of the lesion was performed and the sanguinolent material obtained was submitted to cytology analysis.

The smears stained with Papanicolaou and Leishman stains revealed the presence of a large number of plasma cells of variable sizes with enlarged nuclei, many of them binucleated. Only about 30\% of the leukocytes present corresponded to polymorphonuclear neutrophils and lymphocytes (Figure 2(a)). Aggregation of red blood cells, characterizing rouleaux formation, was also observed (Figure 2(b)).

With the differential diagnosis of MM, the clinical laboratory reported that, despite a borderline concentration of total serum protein of $8.0 \mathrm{~g} / \mathrm{dl}$, electrophoresis

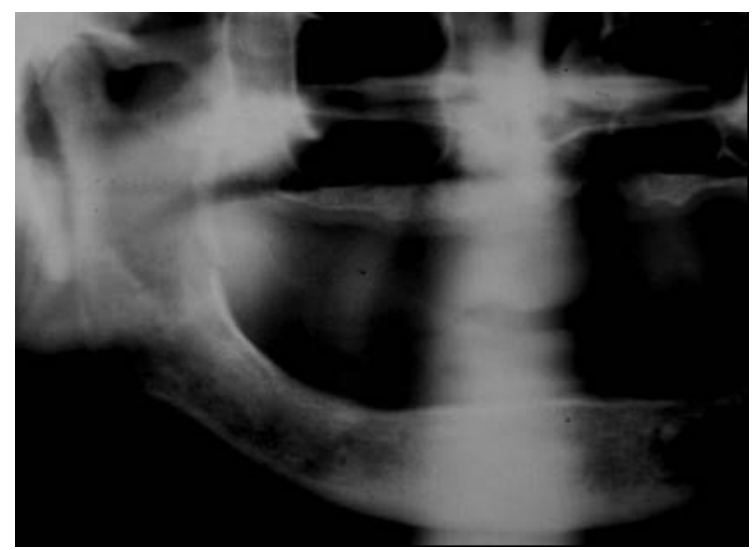

Figure 1. Orthopantomograph showing a transverse fracture in the median portion of the right ascending mandibular ramus, associated with a radiolucent lesion affecting a large portion of this anatomic structure. revealed a monoclonal peak in a band related to gamma globulins, with this peak corresponding to $3.24 \mathrm{~g} / \mathrm{dl}$ (40.5\% of total protein). Urinary analysis was negative for Bence-Jones protein.

Plain radiographs of skull demonstrated the presence of punched-out osteolytic lesions (Figure 3(a)), a sui generis aspect also seen in the vertebra on computed tomography scans of the skeleton (Figure 3(b)).

\section{DISCUSSION}

MM is the third most common form of haematological malignancy after non-Hodgkin’s lymphoma and leukemia [5].

In MM, bone pain is a frequent symptom and is normally associated with pathological fractures, with the skull, vertebrae, ribs and femur being the most affected bones. Oral manifestations of the disease generally in-

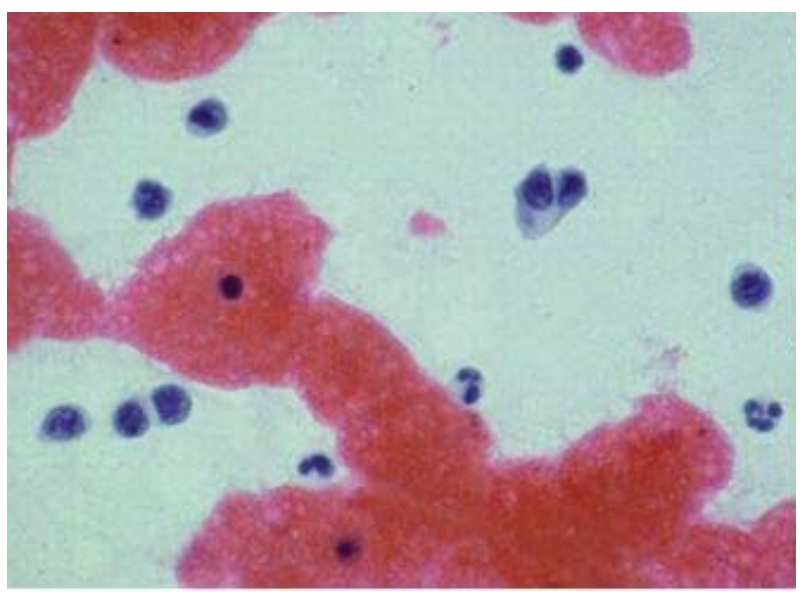

(a)

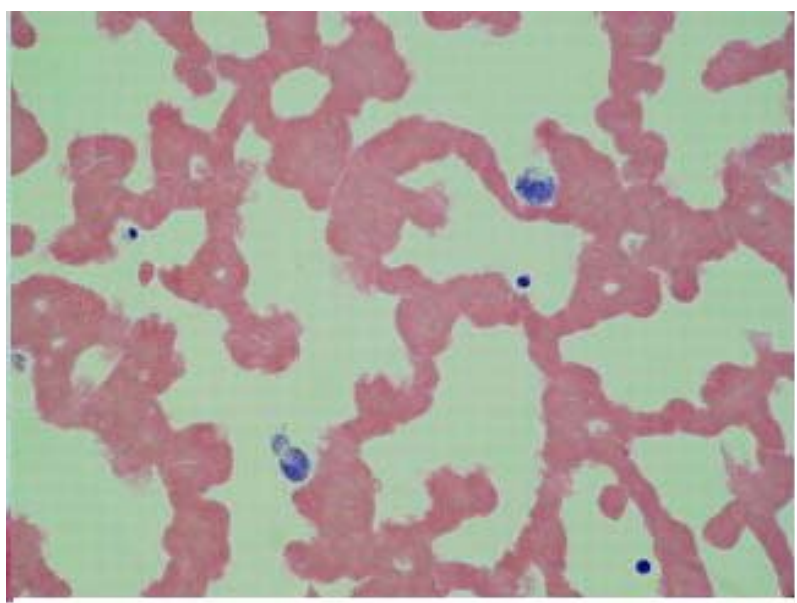

(b)

Figure 2. (a) Smear showing a large number of pleomorphic plasma cells of variables sizes with enlarged nuclei, many of them binucleated. Papanicolaou 630×; (b) Smear showing the aggregation of red blood cells, characterizing rouleaux formation. Papanicolaou 630×. 


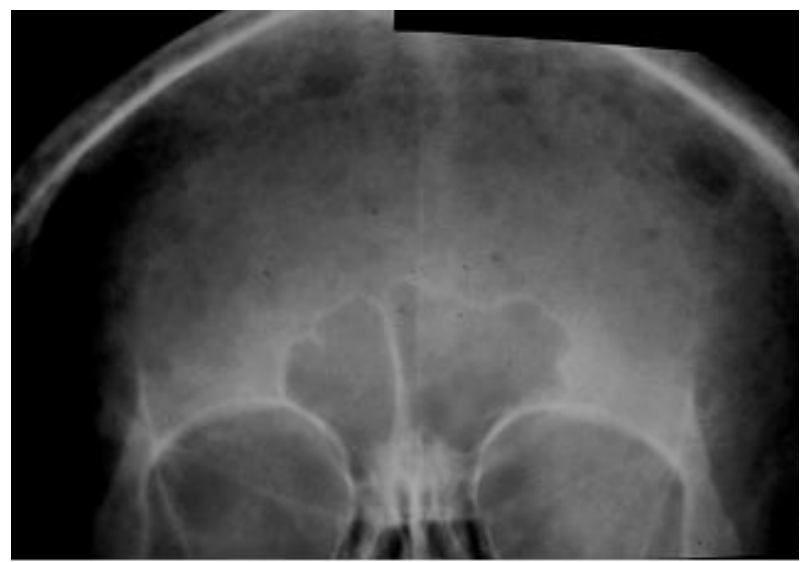

(a)

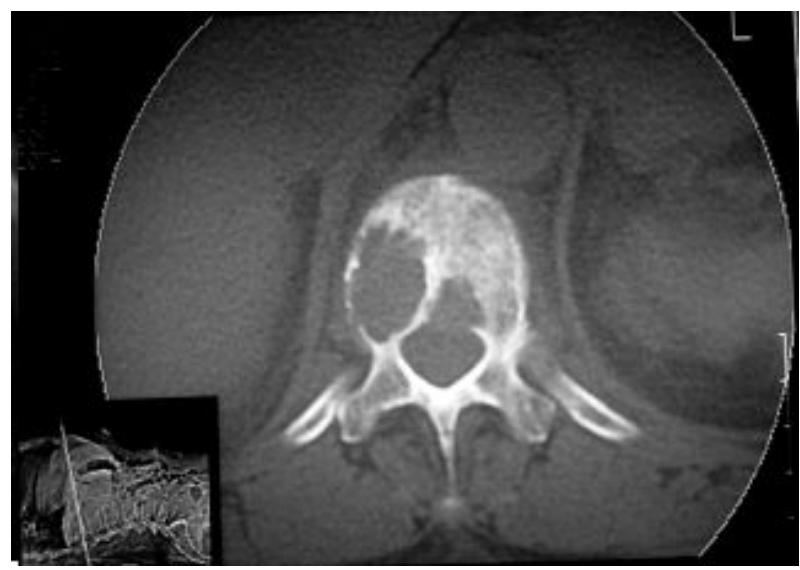

(b)

Figure 3. (a) Anteroposterior radiograph of the skullcap showing diffusely distributed punched-out osteolytic lesions; (b) A punched-out lesion in the vertebra observed on a computed tomography scan of the skeleton.

volve the jaw bones, preferentially the mandible (premolar region, mandibular angle and ascending ramus), and are observed in $25 \%$ of cases. Radiographically, these manifestations present as osteolytic lesions characterized by multiple well- or poorly delimited radiolucent areas, accompanied or not by fractures $[2,5,9]$. In the case of involvement of the jaw bones, expansion of cortical bone might be observed in some cases, with trauma to the mucosal lining of the affected region causing ulceration [8-10]. Other clinical manifestations include tongue numbness or paresthesia, tooth loss or migration and root resorption $[11,12]$.

In $15 \%$ to $25 \%$ of cases, $\mathrm{MM}$ is associated with the deposition of an amyloid substance related to high levels of immunoglobulin light chains, which occurs early during the course of the disease in soft tissues, preferentially the tongue and periorbital region. These lesions manifest as nodules resulting from the deposition of circulating light-chain proteins that, when metabolized by the kidneys, appear in urine and are known as Bence-Jones pro- teins $[4,13]$. This is an important sign which might be the first clinical manifestation of MM.

The present case emphasized the importance of the dentist as a healthcare professional who should be aware of possible systemic diseases that might have repercussions at the maxillomandibular complex. Without knowledge of the disease and being only aware of its oral manifestation, the patient often seeks a dentist who, in turn, should be able to diagnose the disease or refer the patient to a competent diagnostic service.

The importance of cytology, a low cost and easily applied exam, should be emphasized to be used in all materials obtained by aspiration or in imprints of biopsy samples, besides the classic application in smears of epithelial surface lesions. This procedure is available to any healthcare professional and may effectively contribute to the diagnosis. In the present case, FNA cytology analysis was performed, permitting the diagnostic hypothesis of $\mathrm{MM}$ and the request of complementary exams (electrophoretic profile of total serum proteins, plain radiograph of skull, computed tomography scan of the skeleton and scint-scan) which helped confirm the diagnosis of MM.

Although MM is a systemic disease whose treatment is not the competence of the dentist, it is the responsibility of this health professional to perform the most complete clinical exam of the patient, requesting complementary exams related to the disease.

The diagnosis of MM was based on laboratory and radiographic findings. Cytologic analysis of the FNA obtained from the lesion permitted a diagnostic hypothesis of MM.

\section{REFERENCES}

[1] Harousseau, J.L., Moreau, P., Attal, M., Facon, T. and Avet-Loiseau, H. (2005) Stem cell transplantation in multiple myeloma. Best Practice \& Research Clinical Haematology, 18, 603-618. doi:10.1016/j.beha.2005.01.005

[2] Reboiras, L.M.D., Garcia, G.A., Antúnez, L.J., Blanco, C.A., Gándara, V.P. and Gándar, R.J.M. (2001) Anaesthesia of the right lower hemilip as a first manifestation of multiple myeloma. Presentation of a case. Oral Medicine, 6, 168-712.

[3] Barlogie, B., Epstein, J., Selvanayagam, P. and Alexanian, R. (1989) Plasma cell myeloma: New biological insights and advances in therapy. Blood, 73, 865-879.

[4] Viggor, S.F., Frezzini, C., Farthing, P.M., Freeman, C.O., Yeoman, C.M. and Thornhill, M.H. (2009) Amyloidosis: An unusual case of persistent oral ulceration. Oral Surgery Oral Medicine Oral Pathology, 108, e46-e50.

[5] Collins, C.D. (2004) Multiple myeloma. Cancer Imaging, 4, S47-S53. doi:10.1102/1470-7330.2004.0010

[6] Kyle, R.A. and Rajkumar, S.V. (2007) Epidemiology of the plasma-cell disorders. Best Practice \& Research Clinical Haematology, 20, 637-664. 
doi:10.1016/j.beha.2007.08.001

[7] Avilés, A., Nambo, M.J., Neri, N., Castañeda, C., Cleto, S. and Huerta-Guzmán, J. (2007) Antitumor effect of zoledronic acid in previously untreated patients with multiple myeloma. Medical Oncology, 24, 227-230. doi:10.1007/BF02698044

[8] Bensinger, W. (2008) Stem-cell transplantation for multiple myeloma in the era of novel drugs. Journal of Clinical Oncology, 26, 480-492. doi:10.1200/JCO.2007.11.6863

[9] Furutani, M., Ohnishi, M. and Tanaka, Y. (1994) Mandibular involvement in patients with multiple myeloma. Journal of Oral and Maxillofacial Surgery, 52, 23-25. doi:10.1016/0278-2391(94)90007-8

[10] Salisbury, P.L. 3rd and Jacoway, J.R. (1983) Oral amyloidosis: A late complication of multiple myeloma. Oral
Surgery Oral Medicine Oral Pathology, 56, 48-50. doi:10.1016/0030-4220(83)90054-3

[11] Monje, F., Gil-Diez, J.L., Campano, F.J. and Alonso del Hoyo, J.R. (1989) Mandibular lesion as the first evidence of multiple myeloma. Journal of Cranio-Maxillofacial Surgery, 17, 315-317. doi:10.1016/S1010-5182(89)80060-5

[12] Epstein, J.B., Voss, N.J. and Stevenson-Moore, P. (1984) Maxillofacial manifestations of multiple myeloma: An unusual case and review of the literature. Oral Surgery Oral Medicine Oral Pathology, 57, 267-271. doi:10.1016/0030-4220(84)90182-8

[13] Sikkink, L.A. and Ramirez-Alvarado, M. (2008) Biochemical and aggregation analysis of Bence Jones proteins from different light chain diseases. Amyloid, 15, 2939. doi:10.1080/13506120701815324 